\section{A qualidade do atendimento ao parto na rede pública hospitalar em uma capital brasileira: a satisfação das gestantes}

\author{
Quality of care for labor and childbirth in a public \\ hospital network in a Brazilian state capital: \\ patient satisfaction
}

\section{La calidad de la atención al parto dentro de la red pública hospitalaria en una capital brasileña: la satisfacción de las gestantes}

\section{Resumo}

O estudo avaliou a qualidade da atenção ao parto na rede pública hospitalar, na cidade do Recife, Pernambuco, Brasil, segundo satisfação das gestantes, por um estudo transversal de cunho exploratório, em todas as unidades públicas hospitalares da rede, agrupadas em natureza da gestão, pela ausculta de 1.000 gestantes. A qualidade da atenção foi realizada segundo estratos de gestão e dimensões da qualidade: acolhimento; respeito ao direito das pessoas; assistência no pré-natal e parto; e ambiência. A significância dos resultados foi analisada pelo teste qui-quadrado de Pearson e Friedman. Houve alta cobertura do pré-natal, sem vinculação com a assistência ao parto e intensa imigração de partos. O tempo de espera pelo atendimento da equipe foi longo, a proporção de partos vaginais foi superior nas maternidades municipais (80\%), houve em apenas $16 \%$ dos partos o contato pele a pele com o bebê; a amamentação ocorreu em 11\% na sala de parto. Entre as esferas de gestão, o hospital filantrópico teve melhor avaliação. As dimensões avaliadas apresentaram diferenças significativas, tendo maiores satisfações com os aspectos: respeito $(88,2 \%)$, gentileza $(86,7 \%)$, trabalho dos médicos $(85,2 \%)$ e confiança nos profissionais (84,3\%). Enquanto as maiores insatisfações relacionaram-se com: temperatura da enfermaria (62,2\%), possibilidade de fazer reclamações $(48,1 \%)$, quantidade e qualidade das roupas $(49,2 \%)$ e privacidade (43\%). Apesar das conquistas, os achados revelaram a necessidade de reorganização da politica de assistência obstétrica com a regionalização, regulação, consolidação das redes de atenção e intervenções na ambiência, visando consolidar a humanização da atenção.

Avaliação em Saúde; Qualidade da Assistência à Saúde; Parto
Ana Lúcia Andrade da Silva 1 Antonio da Cruz Gouveia Mendes 2 Gabriella Morais Duarte Miranda 3 Wayner Vieira de Souza 2

doi: 10.1590/0102-311X00175116

\author{
Correspondência \\ A. L. A. Silva \\ Centro Acadêmico de Vitória, Universidade Federal de \\ Pernambuco. \\ Rua Alto do Reservatório $s / n$, Vitória de Santo Antão, PE \\ 55608-680, Brasil. \\ anadasilva78@yahoo.com.br \\ 1 Centro Acadêmico de Vitória, Universidade Federal de \\ Pernambuco, Vitória de Santo Antão, Brasil. \\ 2 Instituto Aggeu Magalhães, Fundação Oswaldo Cruz, Recife, \\ Brasil. \\ 3 Departamento de Medicina Social, Universidade Federal de \\ Pernambuco, Recife, Brasil.
}




\section{Introdução}

No Brasil, o percurso da organização da assistência obstétrica determinou a consolidação de forma hegemônica do modelo tecnocrático, excessivamente medicalizado, não baseado em evidências científicas, com a despersonalização do cuidado e o predomínio do uso de tecnologia 1,2.

Seguindo as recomendações da Organização Mundial da Saúde (OMS) para o implemento da humanização da assistência ao parto, foi lançado no Brasil, no ano 2000, o Programa de Humanização no Pré-natal e Nascimento (PHPN) 3. A assistência humanizada ao parto representa a contracultura ao modelo hegemônico e envolve conhecimentos, práticas e atitudes, para promover partos e nascimentos saudáveis. Garantindo a privacidade, a autonomia e o protagonismo da mulher com o desenvolvimento de procedimentos comprovadamente benéficos, sem intervenções desnecessárias, com o respeito às escolhas informadas e a presença de um acompanhante à escolha da parturiente 4,5.

As importantes estratégias desenvolvidas ao longo desses anos não têm sido suficientes para garantir a qualidade da atenção prestada às mulheres, refletindo o efeito limitado das políticas públicas dirigidas à mudança do paradigma de assistência ao parto. Estudos demonstram que o acesso aos serviços obstétricos não é igualitário 6,7, há iniquidade na distribuição de unidades e leitos 8 , inadequações de estrutura das maternidades 9,10 , persistência no desenvolvimento das práticas obstétricas não recomendáveis, tais como uso de ocitocina, episiotomia, manobra de Kristeller, entre outras 4,5,11,12, além do aumento constante das taxas de cesarianas, da prematuridade e do baixo peso ao nascer caracterizando a fragilidade dos cuidados prestados $13,14,15$.

No contexto da avaliação de uma política pública prioritária, a satisfação das usuárias configura-se como importante campo na avaliação da qualidade, na medida em que seu nível de satisfação reflete os diversos momentos do atendimento e a maneira como os cuidados técnicos são dispensados ou recebidos, no relacionamento entre os usuários e o serviço de saúde 16,17,18. Na perspectiva das gestantes, à ambiência das unidades e a relação com os profissionais de saúde, tais como escuta e acolhimento, influenciam no seu julgamento sobre os cuidados recebidos 4,19,20.

Donabedian ${ }^{21}$ propôs considerar os não especialistas (os pacientes) na definição de parâmetros e mensuração da qualidade dos serviços. Para ele, a avaliação do ponto de vista dos usuários é feita, sobretudo, por meio do atributo aceitabilidade, que se refere à conformidade dos serviços oferecidos em relação às expectativas e aspirações dos pacientes e seus familiares. A dimensão da aceitabilidade comporta condições de acessibilidade ao serviço, relação médico-paciente, adequação das dependências e instalações, preferências em relação aos efeitos e custos do tratamento, bem como tudo aquilo que o paciente considera justo ou equânime.

A avaliação da assistência obstétrica pelas gestantes se configura, então, como uma etapa fundamental para mensurar a qualidade da atenção. Nesse contexto, tendo em vista que a avaliação dos serviços de saúde subsidia o planejamento e a gestão do sistema, contribuindo para a melhoria de sua qualidade, este estudo se propôs avaliar a qualidade da atenção ao parto na rede pública hospitalar, na cidade do Recife, Pernambuco, Brasil, mediante a satisfação das suas usuárias.

\section{Métodos}

Foi realizado um estudo quantitativo transversal, de cunho exploratório, sobre a qualidade da assistência ao parto hospitalar na rede pública da cidade do Recife, capital do Estado de Pernambuco, pela avaliação da satisfação das gestantes usuárias do Sistema Único de Saúde (SUS).

Em 2015, na cidade do Recife, a rede pública de atenção ao parto hospitalar era composta por 8 unidades: 3 maternidades municipais de referência ao parto de médio risco; 3 hospitais estaduais; 1 hospital filantrópico e 1 hospital federal, referências ao parto de alto risco para todo o Estado de Pernambuco. Nessa rede, são realizados aproximadamente 27 mil partos ao ano no SUS, representando $63 \%$ e $27 \%$ dos partos ocorridos na Região Metropolitana do Recife e do Estado de Pernambuco, respectivamente.

Foi realizada uma amostra estratificada agrupando-se as oito unidades segundo esfera/natureza de gestão, conformando-se quatro estratos: municipal (3 maternidades), estadual (3 hospitais), filantrópico (1 unidade) e federal (1 unidade). Tomando-se como referência o percentual de satisfação dos 
usuários nordestinos com a assistência recebida no SUS 22, definiu-se a expectativa de $60 \%$ de satisfação das usuárias com a assistência recebida, erro absoluto de $6 \%$ e $5 \%$ de significância estatística. Considerando-se o quantitativo total do número de partos realizados na rede no trimestre (6.720), período estabelecido para realização da coleta dos dados, obteve-se uma amostra de 250 usuárias por estrato, totalizando uma amostra de 1.000 usuárias a serem entrevistadas. Para os estratos compostos por mais de uma unidade, o $\mathrm{N}$ foi distribuído proporcionalmente ao número de partos realizados em cada serviço.

As usuárias foram entrevistadas enquanto estiveram internadas nas unidades, utilizando-se ques-

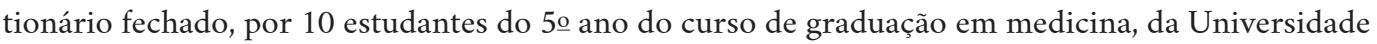
Federal de Pernambuco. As visitas foram realizadas todos os dias da semana, em diversos horários, durante o trimestre estabelecido para a coleta dos dados, quando todas as usuárias foram convidadas a participar. As que concordaram foram submetidas à entrevista, até completar o $\mathrm{N}$ de cada estrato. Durante o processo da coleta dos dados, 43 usuárias não se dispuseram a participar do estudo, representando 4,3\% de recusas. Antes do início da escuta, o Termo de Consentimento Livre e Esclarecido foi apresentado, explicitando que se tratava de pesquisa externa à unidade e que todas as informações colhidas seriam mantidas sob sigilo, sem acesso a qualquer funcionário ou profissional dos serviços.

Foi realizado estudo piloto para avaliação do questionário e treinamento dos estudantes para discussão e apresentação dos objetivos da pesquisa e instrumentos de coleta dos dados. O questionário foi elaborado especificamente para os propósitos desta pesquisa, baseando-se no referencial teórico da avaliação da qualidade dos serviços de saúde de Donabedian 21 e da matriz de avaliação do Projeto de Metodologia de Avaliação do Desempenho do Sistema de Saúde Brasileiro (PROADESS) 23, utilizando-se também as principais políticas que normatizam a assistência obstétrica no SUS 24,25.

O questionário foi composto por 58 questões, distribuídas em quatro seções: (i) identificação do questionário/entrevistador; (ii) caracterização das usuárias; (iii) avaliação da assistência; e (iv) avaliação da satisfação das usuárias. As questões que se referiram à satisfação apresentavam cinco alternativas para julgamento: péssimo, ruim, regular, bom e ótimo.

Com base no referencial adotado, foram definidas quatro dimensões da satisfação: (i) Acolhimento: o tempo de espera para atendimento na recepção, a gentileza dos profissionais na recepção, o atendimento na recepção de forma geral, e o tempo de espera para atendimento pela equipe de saúde; (ii) Respeito ao direito das pessoas: o respeito dos profissionais, a confiança nos profissionais, a privacidade, o apoio nos momentos de dor e medo, a possibilidade de fazer perguntas e receber orientações, e a possibilidade de fazer reclamações; (iii) Assistência: a assistência pré-natal, as orientações dos cuidados com o recém-nascido, o trabalho dos médicos, e o trabalho da equipe de enfermagem; e (iv) Ambiência: a temperatura, o barulho, o conforto, a limpeza, a quantidade e qualidade das refeições, e a quantidade e a qualidade das roupas.

A descrição das usuárias, segundo atributos pessoais e assistenciais foi apresentada em frequências absolutas e relativas. Para verificar diferenças no atendimento prestado entre os estratos das unidades (esferas de gestão/natureza), utilizou-se o teste qui-quadrado de Pearson. Todas as decisões foram tomadas levando em conta o nível de significância estatística de 5\%.

Para garantir a adequada representatividade entre os estratos, procedeu-se à ponderação dos resultados, pelo número de partos realizados em cada estrato: total (6.725); municipal (2.175 partos - 32,4\%); estadual (2.450 partos - 36,4\%); filantrópico (1.600 partos - 23,8\%) e federal (500 partos 7,4\%). Aplicaram-se os respectivos fatores de correção: 1,296, 1,456, 0,952 e 0,296.

O grau de satisfação das participantes foi mensurado sob a hipótese de independência das observações, criou-se uma variável dicotômica, considerando que os atributos péssimo, ruim e regular referiam-se à insatisfação, e bom e ótimo, à satisfação.

Para verificar a existência de diferenças significativas entre as dimensões de insatisfação e seus atributos, optou-se pelo teste de Friedman, por se tratar de amostras relacionadas, as mesmas pessoas avaliando diferentes aspectos. Assim, foram apresentados os means rankings de insatisfação entre as usuárias, considerando quanto menor a média, maior a insatisfação das participantes.

A proporção de insatisfação foi apresentada considerando o número total de usuárias que responderam sobre cada aspecto avaliado, contudo o $\mathrm{N} f$ considerado pelo teste de Friedman representou o conjunto de pessoas que respondeu sobre todos os aspectos. 
A pesquisa é parte dos resultados do projeto de tese de doutorado aprovada pelo Comitê de Ética em Pesquisa do Centro de Pesquisas Aggeu Magalhães, Fundação Oswaldo Cruz (Certificado de Apresentação para Apreciação Ética 13161113.7.0000.5190) e financiado pelo Ministério da Saúde.

\section{Resultados}

Entre as 1.000 usuárias entrevistadas, mais de 21\% eram mães adolescentes, e cerca de 30\% tinham Ensino Fundamental incompleto ou nenhuma escolaridade. A maioria (75,6\%) apresentava entre 20 e 39 anos e cor parda (69,4\%), segundo sua autopercepção, 39,1\% tinham Ensino Médio completo, $61,7 \%$ residiam fora cidade do Recife e cerca de $48 \%$ eram primíparas (Tabela 1).

Mais de 95\% das usuárias realizaram pré-natal, dessas, 56,7\% fizeram 7 ou mais consultas, e a minoria $(25,2 \%)$ recebeu informações sobre o local onde iria parir. As unidades básicas de saúde representaram o principal local para realização dessa assistência, com frequência superior a 70\%. Mais da metade $(56,4 \%)$ das gestantes passou por duas ou mais unidades na busca por atendimento no momento do parto, com $48,7 \%$ de usuárias transferidas entre as maternidades. A ambulância (480) e o carro próprio (383) foram os meios de transportes mais utilizados. Cerca de $28 \%$ das gestantes percorreram mais de $50 \mathrm{~km}$, levando mais de duas horas para chegar à unidade em que receberam atendimento, além disso quase a totalidade (98,9\%) não recebeu auxílio financeiro para o deslocamento (Tabela 2).

Em relação ao atendimento recebido nas unidades, mais de $92 \%$ das gestantes foram atendidas na recepção em até 30 minutos. O tempo de espera pelo atendimento da equipe de saúde apresentou

Tabela 1

Caracterização das usuárias atendidas na rede pública hospitalar de atenção ao parto. Recife, Pernambuco, Brasil, 2015.

\begin{tabular}{|c|c|c|}
\hline Caracterização das usuárias & $\mathbf{n}$ & $\%$ \\
\hline \multicolumn{3}{|l|}{ Faixa etária (anos) } \\
\hline $10-19$ & 215 & 21,5 \\
\hline $20-39$ & 756 & 75,6 \\
\hline 40 e mais & 29 & 2,9 \\
\hline \multicolumn{3}{|l|}{ Cor } \\
\hline Parda/Morena & 694 & 69,4 \\
\hline Negra & 103 & 10,3 \\
\hline Branca & 175 & 17,5 \\
\hline Amarela & 27 & 2,7 \\
\hline Outra & 1 & 0,1 \\
\hline \multicolumn{3}{|l|}{ Escolaridade } \\
\hline Sem escolaridade e Fundamental incompleto & 299 & 29,9 \\
\hline Fundamental completo e Médio incompleto & 310 & 31,0 \\
\hline Médio completo e Superior incompleto & 372 & 37,2 \\
\hline Superior completo e Pós-graduação & 19 & 1,9 \\
\hline \multicolumn{3}{|l|}{ Residência } \\
\hline Recife & 383 & 38,3 \\
\hline Região Metropolitana do Recife & 315 & 31,5 \\
\hline Outras regiões de Pernambuco & 298 & 29,8 \\
\hline Outros estados & 4 & 0,4 \\
\hline \multicolumn{3}{|l|}{ Número de filhos } \\
\hline 1 & 477 & 47,7 \\
\hline 2 & 295 & 29,5 \\
\hline 3 ou mais & 228 & 22,8 \\
\hline
\end{tabular}




\section{Tabela 2}

Descrição das usuárias atendidas na rede pública hospitalar de atenção ao parto, segundo atributos pessoais e assistenciais. Recife, Pernambuco, Brasil, 2015.

\begin{tabular}{|c|c|c|}
\hline Pré-parto & n & $\%$ \\
\hline Realizou o pré-natal & 980 & 98,0 \\
\hline \multicolumn{3}{|l|}{ Sim (local?) } \\
\hline Unidade de Atenção Básica & 695 & 69,5 \\
\hline Unidade de Atenção Básica e outro Serviço de Saúde & 69 & 6,9 \\
\hline Unidade Especializada & 39 & 3,9 \\
\hline Neste hospital & 100 & 10,0 \\
\hline Outro hospital & 77 & 7,7 \\
\hline Não realizou o pré-natal & 20 & 2,0 \\
\hline \multicolumn{3}{|l|}{ Consultas de pré-natal realizadas * } \\
\hline 1 a 3 & 63 & 6,4 \\
\hline 4 a 6 & 359 & 36,6 \\
\hline 7 ou mais & 556 & 56,7 \\
\hline \multicolumn{3}{|l|}{ Recebeu informação sobre o local onde realizaria o parto } \\
\hline Sim & 247 & 25,2 \\
\hline Não & 733 & 74,8 \\
\hline \multicolumn{3}{|l|}{ Unidades por onde passou até o atendimento para o parto } \\
\hline 1 & 436 & 43,6 \\
\hline 2 & 451 & 45,1 \\
\hline 3 ou mais & 113 & 11,3 \\
\hline \multicolumn{3}{|l|}{ Transferência entre unidades de saúde } \\
\hline Sim & 487 & 48,7 \\
\hline Não & 513 & 51,3 \\
\hline \multicolumn{3}{|l|}{ Meio de transporte utilizado } \\
\hline A pé & 12 & 1,2 \\
\hline Ambulância & 480 & 48,0 \\
\hline Carro próprio & 383 & 38,3 \\
\hline Transporte público & 108 & 10,8 \\
\hline Outros & 17 & 1,7 \\
\hline \multicolumn{3}{|l|}{ Tempo gasto para chegar à unidade em que realizou o parto ** } \\
\hline$<30$ minutos & 379 & 37,9 \\
\hline 31 minutos até 1 hora & 176 & 17,6 \\
\hline 1 a 2 horas & 139 & 13,9 \\
\hline$>2$ até 4 horas & 146 & 14,6 \\
\hline 4 horas ou mais & 136 & 13,6 \\
\hline \multicolumn{3}{|c|}{ Distância percorrida entre a residência e a unidade onde realizou o parto (km) } \\
\hline$<15$ & 455 & 45,5 \\
\hline$>15$ até 50 & 264 & 26,4 \\
\hline$>50$ até 100 & 127 & 12,7 \\
\hline$>100$ até 200 & 100 & 10,0 \\
\hline$>200$ & 54 & 5,4 \\
\hline \multicolumn{3}{|l|}{ Recebeu auxílio financeiro para o transporte } \\
\hline $\operatorname{sim}$ & 11 & 1,1 \\
\hline Não & 989 & 98,9 \\
\hline
\end{tabular}

* Entre as 980 usuárias que fizeram pré-natal, 2 (0,2\%) não souberam informar quantas consultas realizaram;

** Entre as 1.000 usuárias entrevistadas, 24 (2,4\%) não souberam responder em quanto tempo chegaram à unidade. 
diferenças significativas entre os estratos $(\mathrm{p}<0,001)$, sendo menor nas unidades estaduais e maior nas municipais e filantrópica (Tabela 3).

\section{Tabela 3}

Caracterização do atendimento recebido pelas usuárias na rede pública hospitalar, segundo as esferas de gestão das unidades. Recife, Pernambuco, Brasil, 2015

\begin{tabular}{|c|c|c|c|c|c|c|c|c|}
\hline \multirow[t]{2}{*}{ Assistência ao parto } & \multicolumn{4}{|c|}{ Estratos (\%) } & \multicolumn{2}{|l|}{ Total } & \multirow[t]{2}{*}{$\chi^{2}$ * } & \multirow[t]{2}{*}{ Valor de $p$ * } \\
\hline & $\begin{array}{l}\text { Municipal } \\
(n=250)\end{array}$ & $\begin{array}{l}\text { Estadual } \\
(n=250)\end{array}$ & $\begin{array}{l}\text { Filantrópico } \\
(n=250)\end{array}$ & $\begin{array}{l}\text { Federal } \\
(n=250)\end{array}$ & $(n=1.000)$ & $\%$ & & \\
\hline $\begin{array}{l}\text { Tempo de espera para receber atendimento } \\
\text { na recepção ** }\end{array}$ & & & & & & & 6,21 & 0,40 \\
\hline Até 30 minutos & 90,3 & 93,0 & 93,1 & 93,1 & 907 & 92,2 & & \\
\hline > 30 minutos a 1 hora & 5,6 & 4,1 & 2,0 & 4,1 & 40 & 4,1 & & \\
\hline$>1$ hora & 4,0 & 2,9 & 4,8 & 2,8 & 37 & 3,7 & & \\
\hline $\begin{array}{l}\text { Tempo de espera para receber atendimento } \\
\text { pela equipe de saúde } \star \star \star\end{array}$ & & & & & & & 57,88 & $<0,001$ \\
\hline Até 30 minutos & 58,0 & 83,8 & 54,6 & 65,6 & 659 & 67,0 & & \\
\hline > 30 minutos a 1 hora & 16,0 & 7,5 & 17,7 & 11,5 & 128 & 13,0 & & \\
\hline$>1$ hora a 2 horas & 14,4 & 3,3 & 13,3 & 11,9 & 98 & 10,0 & & \\
\hline$>2$ horas & 11,6 & 5,4 & 14,5 & 11,1 & 99 & 10,0 & & \\
\hline Tipo de parto realizado & & & & & & & 97,56 & $<0,001$ \\
\hline Vaginal & 80,0 & 39,6 & 44,8 & 55,2 & 551 & 55,1 & & \\
\hline Cesariana & 20,0 & 60,4 & 55,2 & 44,8 & 449 & 44,9 & & \\
\hline Profissional que realizou o parto \# & & & & & & & 5,81 & 0,12 \\
\hline Médico & 86,5 & 92,4 & 92,3 & 90,4 & 823 & 90,4 & & \\
\hline Enfermeiro & 13,5 & 7,6 & 7,7 & 9,6 & 88 & 9,6 & & \\
\hline Permissão da presença do acompanhante \#\# & & & & & & & 96,59 & $<0,001$ \\
\hline Em alguns momentos & 40,7 & 61,4 & 18,4 & 38,9 & 435 & 43,3 & & \\
\hline Em todos os momentos & 59,3 & 38,6 & 81,6 & 61,1 & 569 & 56,7 & & \\
\hline $\begin{array}{l}\text { Contato pele a pele com bebê na sala } \\
\text { de parto }\end{array}$ & & & & & & & 5,41 & 0,14 \\
\hline Sim & 80,4 & 83,6 & 84,0 & 88,0 & 830 & 83,0 & & \\
\hline Não & 19,6 & 16,4 & 16,0 & 12,0 & 170 & 17,0 & & \\
\hline Amamentação na sala de parto & & & & & & & 42,58 & $<0,001$ \\
\hline $\operatorname{sim}$ & 6,0 & 9,2 & 10,0 & 22,0 & 93 & 9,3 & & \\
\hline Não & 93,6 & 87,6 & 88,4 & 75,2 & 888 & 88,8 & & \\
\hline Contraindicação clínica & 0,4 & 3,2 & 1,6 & 2,8 & 19 & 1,9 & & \\
\hline $\begin{array}{l}\text { Necessidade de cuidado hospitalar aos } \\
\text { recém-nascidos }\end{array}$ & & & & & & & 152,20 & $<0,001$ \\
\hline Não & 79,6 & 51,6 & 77,6 & 64,8 & 678 & 67,8 & & \\
\hline Sim & 20,4 & 48,4 & 22,4 & 35,2 & 322 & 32,2 & & \\
\hline UTI & 3,9 & 50,4 & 42,9 & 12,5 & 118 & 11,8 & & \\
\hline Incubadora & 41,2 & 24,8 & 28,6 & 10,2 & 89 & 8,9 & & \\
\hline Berço aquecido & 54,9 & 24,8 & 28,6 & 77,3 & 115 & 11,5 & & \\
\hline Internamento em alojamento conjunto \#\#\# & & & & & & & 44,66 & $<0,001$ \\
\hline $\operatorname{sim}$ & 92,0 & 77,2 & 87,6 & 71,6 & 841 & 84,1 & & \\
\hline Não & 8,0 & 22,8 & 12,4 & 28,4 & 159 & 15,9 & & \\
\hline Orientação e estímulo para amamentação & & & & & & & 13,06 & 0,04 \\
\hline Sim & 83,2 & 82,8 & 91,6 & 88,0 & 858 & 85,8 & & \\
\hline Não & 16,8 & 16,8 & 7,6 & 11,6 & 142 & 14,2 & & \\
\hline
\end{tabular}

(continua) 
Tabela 3 (continuação)

\begin{tabular}{|c|c|c|c|c|c|c|c|c|}
\hline \multirow[t]{2}{*}{ Assistência ao parto } & \multicolumn{4}{|c|}{ Estratos (\%) } & \multicolumn{2}{|l|}{ Total } & \multirow[t]{2}{*}{$\chi^{2 *}$} & \multirow[t]{2}{*}{ Valor de $p$ * } \\
\hline & $\begin{array}{c}\text { Municipal } \\
(n=250)\end{array}$ & $\begin{array}{l}\text { Estadual } \\
(n=250)\end{array}$ & $\begin{array}{l}\text { Filantrópico } \\
\qquad(n=250)\end{array}$ & $\begin{array}{l}\text { Federal } \\
(n=250)\end{array}$ & $(n=1.000)$ & $\%$ & & \\
\hline Orientação sobre os cuidados com o bebê & & & & & & & 6,21 & 0,40 \\
\hline Sim & 66,8 & 78,8 & 94,4 & 86,0 & 792 & 79,2 & & \\
\hline Não & 33,2 & 21,2 & 5,6 & 14,0 & 208 & 20,8 & & \\
\hline Orientação sobre os cuidados puerperais & & & & & & & 39,07 & $<0,001$ \\
\hline Sim & 53,6 & 68,0 & 73,6 & 78,0 & 654 & 65,4 & & \\
\hline Não & 46,4 & 32,0 & 26,4 & 22,0 & 346 & 34,6 & & \\
\hline Sofreu violência durante o atendimento & & & & & & & 7,16 & 0,07 \\
\hline Sim & 7,6 & 5,2 & 2,4 & 4,8 & 53 & 5,3 & & \\
\hline Não & 92,4 & 94,8 & 97,6 & 95,2 & 947 & 94,7 & & \\
\hline Escolha da unidade para atendimento futuro & & & & & & & 33,45 & $<0,001$ \\
\hline $\operatorname{Sim}$ & 66,8 & 73,6 & 87,6 & 80,0 & 752 & 75,2 & & \\
\hline Não & 33,2 & 26,4 & 12,4 & 20,0 & 248 & 24,8 & & \\
\hline
\end{tabular}

* Resultados do teste de qui-quadrado de Pearson;

** Entre as 1.000 usuárias entrevistadas: 15 (1,5\%) não souberam responder quanto tempo esperaram para serem atendidas na recepção;

*** $16(1,6 \%)$ usuárias não souberam responder quanto esperaram até serem atendidas pela equipe de saúde;

\# 92 (9,2\%) usuárias não souberam responder qual profissional realizou seu parto;

\#\# 17 (1,7\%) usuárias não souberam responder sobre a permissão da presença de acompanhante durante o internamento;

\#\#\# 6 (0,6\%) usuárias não souberam responder sobre o internamento em alojamento conjunto.

A proporção de partos vaginais foi superior e significativa entre as unidades municipais (80\%), enquanto nos hospitais estaduais e filantrópico os partos cirúrgicos ultrapassaram $55 \%$. Em todas as unidades, a maioria dos partos foi realizada por médicos. Nas unidades municipais, observou-se a maior proporção $(13,5 \%)$ de partos realizados por enfermeiros. A permissão da presença do acompanhante em todos os momentos foi maior no hospital filantrópico e menor nas maternidades estaduais $(\mathrm{p}<0,001)$ (Tabela 3).

O contato pele a pele na sala de parto foi superior a $80 \%$ em todas as unidades. Contrariamente, a amamentação na sala de parto apresentou baixas proporções em toda a rede, entretanto com diferenças significativas, sendo superior na unidade federal (22\%) e inferior nas municipais (6\%). Mais de $30 \%$ dos recém-nascidos necessitaram de cuidados hospitalares, dentre eles, a maioria esteve em UTI nas instituições estaduais $(50,4 \%)$ e filantrópica $(42,9 \%)$. O berço aquecido foi o equipamento mais utilizado nas unidades federal (77,3\%) e municipais (54,9\%). O internamento em alojamento conjunto foi superior nas unidades municipais e inferior nos internamentos dos hospitais federal e estaduais $(\mathrm{p}<0,001)$.

Em relação à orientação e ao estímulo à amamentação, verificou-se a maior proporção (91,6\%) no hospital filantrópico. Contudo, mais de $14 \%$ das parturientes não foram abordadas sobre a questão nas outras unidades da rede. Os menores percentuais de orientação sobre os cuidados puerperais e com os recém-nascidos foram verificados nas unidades municipais e estaduais ( $p<0,001$ ) (Tabela 3).

Entre as usuárias entrevistadas, 5,3\% relataram ter sofrido violência durante o atendimento, com maiores proporções nas unidades municipais e estaduais.

A avaliação geral com o atendimento recebido apresentou diferenças entre os estratos, sendo maior a satisfação das usuárias com o atendimento no hospital filantrópico, e menor entre as gestantes assistidas nas unidades municipais e estaduais ( $\mathrm{p}<0,001)$ (Tabela 3 ).

A análise da satisfação das usuárias apresentou diferenças em todas as dimensões avaliadas ( $\mathrm{p}<$ 0,001). As usuárias estiveram mais satisfeitas com a Assistência e o Acolhimento e mais insatisfeitas com a Ambiência (47,1\%) e o Respeito ao direito das pessoas (29,7\%) (Tabela 4).

Em relação ao Acolhimento, a maior satisfação foi com a gentileza dos profissionais $(85,7 \%)$ e as maiores insatisfações foram com o tempo de espera para atendimento pela equipe de saúde $(41,8 \%)$, 


\section{Tabela 4}

Insatisfação das usuárias com a qualidade da assistência ao parto, na rede pública hospitalar. Recife, Pernambuco, Brasil, 2015.

\begin{tabular}{|c|c|c|c|c|c|c|c|}
\hline \multirow[t]{2}{*}{ Dimensões da satisfação } & \multirow[t]{2}{*}{ Mean ranking } & \multirow[t]{2}{*}{ Respostas * } & \multicolumn{2}{|c|}{ Insatisfação } & \multirow[t]{2}{*}{$\chi^{2 * *}$} & \multirow[t]{2}{*}{$\mathrm{DF} * *$} & \multirow[t]{2}{*}{ Valor de $p$ ** } \\
\hline & & & 1 & $\%$ & & & \\
\hline Acolhimento & 2,58 & 914 & 222 & 24,3 & & & \\
\hline Respeito ao direito das pessoas & 2,55 & 953 & 283 & 29,7 & & & \\
\hline Assistência no pré-natal, parto e pós-parto & 2,70 & 942 & 206 & 21,9 & 545,0 & 3 & $<0,001$ \\
\hline Ambiência & 2,17 & 981 & 462 & 47,1 & & & \\
\hline Acolhimento & $\mathrm{N} f=880 * \star \star$ & & & & 371,7 & 3 & $<0,001$ \\
\hline Tempo de espera para atendimento na recepção & 2,46 & 918 & 213 & 23,2 & & & \\
\hline Gentileza dos profissionais na recepção & 2,79 & 881 & 126 & 14,3 & & & \\
\hline Atendimento na recepção de maneira geral & 2,73 & 892 & 145 & 16,2 & & & \\
\hline Tempo de espera para atendimento pela equipe & 2,02 & 964 & 403 & 41,8 & & & \\
\hline Respeito ao direito das pessoas & $N f=729 * \star \star$ & & & & 795,6 & 5 & $<0,001$ \\
\hline Respeito dos profissionais & 4,27 & 997 & 136 & 13,7 & & & \\
\hline Confiança nos profissionais & 4,08 & 997 & 178 & 17,9 & & & \\
\hline Privacidade & 2,59 & 998 & 480 & 48,1 & & & \\
\hline Apoio em momentos de dor e medo & 3,85 & 997 & 234 & 23,5 & & & \\
\hline Possibilidade em fazer perguntas e receber orientações & 3,51 & 965 & 287 & 29,8 & & & \\
\hline Possibilidade em fazer reclamações & 2,70 & 765 & 381 & 49,8 & & & \\
\hline Assistência no pré-natal, parto e pós-parto & $\mathrm{N} f=799 * \star \star$ & & & & 138,1 & 3 & $<0,001$ \\
\hline Assistência pré-natal & 2,25 & 997 & 268 & 26,9 & & & \\
\hline Orientações dos cuidados com o recém-nascido & 2,37 & 792 & 150 & 19,0 & & & \\
\hline Trabalho dos profissionais médicos & 2,78 & 1000 & 175 & 17,5 & & & \\
\hline Trabalho da equipe de enfermagem & 2,60 & 1000 & 231 & 23,1 & & & \\
\hline Ambiência & $N f=879 * \star \star$ & & & & 284,1 & 5 & $<0,001$ \\
\hline Temperatura na enfermaria & 2,87 & 1000 & 583 & 58,3 & & & \\
\hline Barulho na enfermaria & 3,48 & 997 & 480 & 48,1 & & & \\
\hline Conforto na enfermaria & 3,48 & 999 & 514 & 51,4 & & & \\
\hline Limpeza na enfermaria & 3,91 & 997 & 393 & 39,4 & & & \\
\hline Quantidade e qualidade das refeições & 3,97 & 993 & 350 & 35,3 & & & \\
\hline Quantidade e qualidade das roupas & 3,29 & 898 & 452 & 50,3 & & & \\
\hline
\end{tabular}

* Total de usuárias que responderam a cada pergunta;

** Resultados do teste de Friedman;

*** Para o teste de Friedman, o n corresponde ao número de usuárias que respondeu sobre todos os aspectos avaliados.

seguidas pelo tempo de espera para atendimento na recepção $(23,2 \%)$. Na avaliação do Respeito ao direito das pessoas, as usuárias estiveram mais satisfeitas com o respeito $(86,3 \%)$ e a confiança $(82,1 \%)$ nos profissionais e mais insatisfeitas com a possibilidade de fazer reclamações $(49,8 \%)$ e com a privacidade $(48,1 \%)$ (Tabela 4$)$.

Em relação aos aspectos avaliados na dimensão de Assistência pré-natal e parto, constatou-se a maior satisfação com o trabalho dos médicos e a menor com a assistência pré-natal. Na dimensão da Ambiência, identificaram-se os maiores percentuais de insatisfação em todos os aspectos avaliados, sendo superior na temperatura da enfermaria $(58,3 \%)$ e conforto $(51,4 \%)$ (Tabela 4$)$. 


\section{Discussão}

O perfil das usuárias atendidas na rede pública de atenção ao parto hospitalar, na cidade do Recife, é de primíparas pardas, com idade entre 20 e 39 anos, com Ensino Médio completo, residentes de Recife e municípios da Região Metropolitana.

Apesar da redução nas taxas de fecundidade e da melhoria das condições sociais ocorridas no Brasil, nas últimas décadas 26, identificou-se que, em Recife, para cada cinco partos realizados, um foi em adolescentes.

Os resultados demonstraram que a maioria das usuárias atendidas na rede reside fora da cidade do Recife, caracterizando uma migração das gestantes para parir na capital. Fluxo em parte justificado porque, entre as oito unidades avaliadas, cinco são hospitais de referência ao parto de alto risco para todo o Estado de Pernambuco, embora mais da metade tenha sido de partos vaginais.

Situação diferente é encontrada no Ceará, onde a assistência ao parto de baixo e médio risco é garantida nos municípios de residência das gestantes, sendo, portanto, a maioria dos partos realizada na capital de residentes de Fortaleza 27.

Os achados da alta adesão e cobertura do pré-natal com a baixa vinculação ao parto revelam a fragmentação no atendimento às gestantes que percorrem por conta própria fluxos desordenados entre as cidades e as maternidades, peregrinando por duas ou mais unidades na busca por atendimento no momento do parto como fica evidenciado nos resultados. Além disso, não foi garantido às gestantes o auxílio financeiro ao deslocamento para realização das consultas de pré-natal e parto.

A falta de conexão entre os serviços que prestam assistência pré-natal e aqueles que disponibilizam assistência ao parto, associada a problemas na organização dos serviços de saúde, à carência e má distribuição de vagas e leitos obstétricos representa um obstáculo entre a busca e a obtenção dos serviços para as gestantes no SUS 28,29.

Diante disso, a Rede Cegonha preconiza que seja concedido apoio financeiro ao deslocamento das gestantes para a realização das consultas de pré-natal e para o local em que será realizado o parto, visando à garantia da qualidade do pré-natal com início precoce e número adequado de consultas e exames, bem como o direito a partos humanizados e seguros 24 .

Verificou-se que as usuárias foram atendidas de imediato na recepção das unidades, todavia houve demora no atendimento pela equipe de saúde, especialmente nas unidades municipais e filantrópica. A permissão da presença do acompanhante em todos os momentos foi assegurada no hospital filantrópico e não garantida nas outras unidades, caracterizando a inadequação da assistência prestada na rede pública do Recife.

A assistência de qualidade ao parto, incluindo os direitos reprodutivos da mulher de ter um acompanhante de sua livre escolha, uma vaga assegurada em maternidade de fácil acesso e atendimento digno (sem espera longa) ocorre em sinergia negativa com a escolaridade, raça e situação socioeconômica das mães 19,30,31.

Os elevados percentuais de partos cesáreos encontrados nas unidades de alta complexidade no Recife refletem a concentração dos serviços de referência na capital. Em um estado de longa extensão territorial, a má distribuição dos serviços e leitos obstétricos configura-se como uma barreira ao acesso das mulheres aos serviços de saúde, forçando-as a percorrer longas distâncias por atendimento, contribuindo sobremaneira para a elevada peregrinação encontrada. As proporções de cesarianas encontradas, muito superiores aos 15\% recomendáveis pela OMS, revelam o caráter intervencionista e medicalizado da atenção ao parto no Brasil, onde o modelo tecnocrático da assistência obstétrica possibilita a utilização acrítica de novas tecnologias e incorporação de grande número de intervenções (muitas vezes desnecessárias), não considerando as mulheres em sua totalidade física, psíquica, afetiva e seus valores $32,33,34$.

Para Leal et al. 6, no Brasil, a alta cobertura do atendimento hospitalar na assistência ao parto é ainda permeada com obstáculos no acesso à maternidade. Ocorre desorganização do sistema de saúde na oferta dos leitos obstétricos e neonatais, com maior concentração da oferta nas capitais dos estados, onde se concentram os serviços com maiores recursos tecnológicos.

Nas maternidades municipais, o quantitativo de partos vaginais esteve adequado ao seu nível de complexidade, com maior participação de enfermeiros, porém essa condição não propiciou a ama- 
mentação na sala de parto, que foi pouco praticada em toda a rede. Em contrapartida, a maioria das mulheres foi sensibilizada e estimulada a amamentar.

Entretanto, um número razoável de mulheres deixou os serviços de saúde sem qualquer abordagem a esse respeito. Achados não condizentes com a proposta da humanização da assistência ao parto, em todas as unidades certificadas em Iniciativa Hospital Amigo da Criança, resultados semelhantes foram verificados por outros estudos 35,36 .

Em relação ao contato pele a pele mãe-bebê e à internação em alojamento conjunto, verificou-se que ambos estiveram instituídos nas unidades, permitindo o fortalecimento dos vínculos às puérperas e seus recém-nascidos.

Nas unidades avaliadas, cinquenta mulheres ouvidas ainda durante o internamento relataram ter sofrido violência, realidade que vem sendo amplamente discutida na literatura 19,37, buscando-se a visibilidade a fim de garantir o respeito à dignidade e aos direitos das mulheres.

$\mathrm{O}$ atendimento nos hospitais filantrópico e federal foi mais bem avaliado pelas usuárias, enquanto os cuidados prestados nas maternidades municipais e estaduais apresentaram insatisfação acima da esperada. Situação que se reveste de importância para a gestão e a organização dos serviços de atenção ao parto na capital.

Em relação à avaliação da satisfação das usuárias, segundo as dimensões da qualidade da assistência adotadas por este estudo, observou-se que as maiores satisfações foram com a Assistência e o Acolhimento, sendo, portanto, as maiores insatisfações com a Ambiência e o Respeito ao direito das pessoas.

$\mathrm{Na}$ dimensão Acolhimento, apesar da boa avaliação com a gentileza e seu atendimento na recepção, aconteceu alto grau de insatisfação com o tempo de espera para atendimento pela equipe de saúde, demonstrando que receber as gestantes de forma inclusiva e responsável requer mais que cortesia e gentileza. A demora pela assistência dos profissionais possivelmente está associada à demanda maior que a capacidade de atendimento nas unidades e ao seu quantitativo de profissionais, condição que interfere na qualidade do atendimento às gestantes nas unidades.

A avaliação dos aspectos relacionados com o Respeito ao direito das pessoas demonstrou alta proporção de satisfação com o respeito e confiança nos profissionais, retratando a capacidade dos serviços de saúde de atender às necessidades das gestantes na perspectiva dos direitos de cidadania. Situação reforçada quando a maioria das gestantes relatou receber apoio em seus momentos de dor e medo. Houve grande insatisfação com a privacidade e impossibilidade de fazer reclamações, o que evidenciou problemas de adequação da estrutura hospitalar. Outros estudos também identificaram avaliações positivas das mulheres relacionadas ao respeito, à confiança e ao trabalho dos profissionais $4,29,38$.

$\mathrm{Na}$ avaliação da Assistência, chama a atenção a alta proporção de satisfação com o trabalho dos médicos, condizente com o sentimento de respeito e confiança verificados na dimensão anterior. Embora a avaliação da assistência pré-natal tenha demonstrado que a maioria das usuárias realizou sete consultas ou mais nas unidades básicas de saúde, a grande insatisfação das usuárias com o prénatal pode estar relacionada à desvinculação entre o pré-natal e o parto, problema ainda não superado nos serviços de saúde do SUS 6,30,33.

Os aspectos avaliados na dimensão Ambiência apresentaram grande insatisfação com a temperatura na enfermaria, o conforto, a qualidade e quantidade das roupas e o barulho, demonstrando que a estrutura das unidades não está adequada à humanização e qualidade da assistência.

Os resultados apontam para avanços na prestação dos cuidados às gestantes e puérperas como a ampliação da cobertura da assistência pré-natal com 7 ou mais consultas, o desenvolvimento de práticas recomendadas pelo modelo humanístico de atenção ao parto, consolidação do acolhimento, e do vínculo entre profissionais e usuárias no sentido do respeito, dignidade e cortesia.

Apesar das conquistas, os achados deste estudo revelaram a necessidade de reorganização da política de assistência obstétrica no Recife e consequentemente no Estado de Pernambuco, na perspectiva da retomada e fortalecimento do processo de regionalização no estado, consolidação das redes de atenção, regulação do acesso e definição de fluxos de referência e contrarreferência, buscando-se superar a peregrinação, longos deslocamentos na busca por atendimento no momento do parto, a desconexão entre a assistência pré-natal e a má distribuição dos serviços e leitos, garantindo o acesso oportuno e equânime aos serviços. 
No que se refere à assistência prestada nas unidades avaliadas, fazem-se necessárias adequações da estrutura física enquanto compromisso com a ambiência, proporcionando o bem-estar das mulheres, bem como preservando o direito à sua voz. Intervenções de resolução em curto prazo e que não requerem grandes aportes de investimentos tais como ajustes na temperatura, ruídos, qualidade das roupas, refeições, privacidade dos alojamentos conjuntos e acesso à ouvidoria, devem ser implementadas com intuito de atender às necessidades das usuárias.

A garantia do atendimento com qualidade requer o fortalecimento das conquistas alcançadas e a consolidação da política de humanização da atenção obstétrica, assegurando às mulheres e aos recémnascidos partos e nascimentos seguros e saudáveis na perspectiva dos direitos de cidadania.

As puérperas foram ouvidas enquanto estavam internadas nas unidades, situação que pode, em parte, ter causado receios no julgamento dos aspectos avaliados, mas a riqueza dos resultados identificados demonstraram a validade e pertinência da abordagem e metodologia adotadas neste estudo. Apesar da importância dos achados, obtidos pela ausculta direta das usuárias, o desenvolvimento de outros estudos que utilizem outras metodologias possibilitarão trazer outras compreensões, ampliando a percepção de tema tão complexo.

\section{Colaboradores}

A. L. A. Silva, A. C. G. Mendes e G. M. D. Miranda contribuíram com a concepção e projeto, análise e interpretação dos dados, redação do artigo, revisão crítica relevante do conteúdo intelectual e aprovação final da versão a ser publicada. W. V. Souza contribuiu com a análise e interpretação dos dados, revisão crítica relevante do conteúdo intelectual e aprovação final da versão a ser publicada. Todos os autores são responsáveis por todos os aspectos do trabalho na garantia da exatidão e integridade de qualquer parte da obra.

\section{Agradecimentos}

Agradecimento ao Ministério da Saúde (Secretaria de Vigilância à Saúde, Edital 20/2013) pelo financiamento da pesquisa. 


\section{Referências}

1. Portella MO. Avanços e incoerências nas políticas de humanização do parto e nascimento. In: Martins PH, Falangola A, Silva AS, Sousa IC, organizadores. Produtivismo na saúde: desafios do SUS na invenção da gestão democrática. Recife: Editora da Universidade Federal de Pernambuco; 2014. p. 119-44.

2. Maia MB. Humanização do parto: política pública, comportamento organizacional e ethos profissional. Rio de Janeiro: Editora Fiocruz; 2010.

3. Diniz CSG. Humanização da assistência ao parto no Brasil: os muitos sentidos de um movimento. Ciênc Saúde Coletiva 2005; 10: 627-37.

4. Bruggemann OM, Monticelli M, Furtado C, Fernandes CM, Lemos FN, Gayeski ME. Filosofia assistencial de uma maternidade-escola: fatores associados à satisfação das mulheres usuárias. Texto Contexto Enferm 2011; 20:658-68

5. Leal MC, Pereira APE, Domingues RMSM, Theme Filha MM, Dias MAB, Nakamura-Pereira $\mathrm{M}$, et al. Intervenções obstétricas durante o trabalho de parto e parto em mulheres brasileiras de risco habitual. Cad Saúde Pública 2014; 30 Suppl:S17-47.

6. Leal MC, Theme-Filha MM, Moura EC, Cecatti JG, Santos LMP. Atenção ao pré-natal e parto em mulheres usuárias do sistema público de saúde residentes na Amazônia Legal e no Nordeste, Brasil 2010. Rev Bras Saúde Matern Infant 2015; 15:91-104.

7. Almeida WS, Szwarcwald CL. Mortalidade infantil e acesso geográfico ao parto nos municípios brasileiros. Rev Saúde Pública 2012; 46:68-76.

8. Campos TP, Carvalho MS. Assistência ao parto no Município do Rio de Janeiro: perfil das maternidades e o acesso da clientela. Cad Saúde Pública 2000; 16:411-20.

9. Bittencourt SDA, Reis LGC, Ramos MM, Rattner D, Rodrigues PL, Neves DCO, et al. Estrutura das maternidades: aspectos relevantes para a qualidade da atenção ao parto e nascimento. Cad Saúde Pública 2014; 30 Suppl:S208-19.

10. Alves MTSSB, Araújo TVB, Alves SV, Marinho LFB, Schiavo L, Menezes G, et al. Atenção ao aborto no Sistema Único de Saúde no Nordeste Brasileiro: a estrutura dos serviços. Rev Bras Saúde Matern Infant 2014; 14:229-39.

11. Apolinário D, Rabelo M, Wolff LDG, Rossi SR, Souza K, Leal GCG. Práticas na atenção ao parto e nascimento sob a perspectiva das puérperas. Rev RENE 2016; 17:20-8.

12. Giglio MRP, França E, Lamounier JA. Avaliação da qualidade da assistência ao parto normal. Rev Bras Ginecol Obstet 2011; 33: 297-304.

13. Silva ALA, Mendes ACG, Miranda GMD, Santos Neto PM. Childbirth care in Brazil: a critical situation has not yet been overcome, 19992013. Rev Bras Saúde Matern Infant 2016; 16:129-48.
14. Domingues RMSM, Dias MAB, Nakamura -Pereira M, Torres JA, d'Orsi E, Pereira APE, et al. Processo de decisão pelo tipo de parto no Brasil: da preferência inicial das mulheres à via de parto final. Cad Saúde Pública 2014; 30 Suppl:S101-16.

15. Santos HG, Andrade SM, Silva AMR, Mathias TAF, Ferrari LL, Mesas AE. Mortes infantis evitáveis por intervenções do Sistema Único de Saúde: comparação de duas coortes de nascimentos. Ciênc Saúde Coletiva 2014; 19 : 907-16.

16. Esperidião MA, Trad LAB. Avaliação de satisfação de usuários: considerações teórico-conceituais. Cad Saúde Pública 2006; 22:1267-76.

17. Jorge MSB, Guimarães JMX, Vieira LB, Paiva FDS, Silva DR, Pinto AGA. Avaliação da qualidade do PSF no Ceará: a satisfação dos usuários. Rev Baiana Saúde Pública 2007; 31:256-66.

18. Vaitsman J, Andrade GRB. Satisfação e responsividade: formas de medir a qualidade e a humanização da assistência à saúde. Ciênc Saúde Coletiva 2005; 10:599-613.

19. d'Orsi E, Brüggemann OM, Diniz CSG, Aguiar JM, Gusman CR, Torres JA, et al. Desigualdades sociais e satisfação das mulheres com o atendimento ao parto no Brasil: estudo nacional de base hospitalar. Cad Saúde Pública 2014; 30 Suppl 1:S154-68

20. Domingues RMSM, Santos EM, Leal MC. Aspectos da satisfação das mulheres com a assistência ao parto: contribuição para o debate. Cad Saúde Pública 2004; 20 Suppl 1:S52-62.

21. Donabedian A. The seven pillars of quality. Arch Pathol Lab Med 1990; 114:1115-8.

22. Campozana GG, Souza WVS, Luna CF, Souza Junior PRB, Szwarcwald CL. Satisfação dos usuários do sistema de saúde brasileiro: fatores associados e diferenças regionais. Rev Bras Epidemiol 2009; 12:281-96.

23. Fundação Oswaldo Cruz. Avaliação de Desempenho do Sistema de Saúde Brasileiro. http:// www.proadess.icict.fiocruz.br/index.php?pa $\mathrm{g}=$ produ (acessado em 02/Set/2016).

24. Secretaria de Atenção à Saúde, Ministério da Saúde. Manual prático da Rede Cegonha. Brasília: Ministério da Saúde; 2011.

25. Secretaria Executiva, Ministério da Saúde. Programa de Humanização no Pré-natal e Nascimento. Brasília: Ministério da Saúde; 2002.

26. Mendes ACG, Sá DA, Miranda GMD, Lyra TM, Tavares RAW. Assistência pública de saúde no contexto da transição demográfica brasileira: exigências atuais e futuras. Cad Saúde Pública 2012; 28:955-64.

27. Queiroz MVO, Jorge MSB, Marques JF, Cavalcante AM, Moreira KAP. Indicadores de qualidade da assistência ao nascimento baseados na satisfação de puérperas. Texto Contexto Enferm 2007; 16:479-87.

28. Viellas EF, Domingues RMSM, Dias MAB, Gama SGN, Theme Filha MM, Costa JV, et al. Assistência pré-natal no Brasil. Cad Saúde Pública 2014; 30 Suppl:S85-100. 
29. Cunha SF, D’Eça Júnior A, Rios ATF, Pestana AL, Mochel EG, Paiva SS. Peregrinação no anteparto em São Luís - Maranhão. Cogitare Enferm 2010; 15:441-7.

30. Matar LD, Diniz CSG. Hierarquias reprodutivas: maternidade e desigualdades no exercício de direitos humanos pelas mulheres. Interface Comun Saúde Educ 2012; 16:107-19.

31. Nahagama EEI, Santiago SN. Parto humanizado e tipo de parto: avaliação da assistência oferecida pelo Sistema Único de Saúde em uma cidade do Sul do Brasil. Rev Bras Saúde Matern Infant 2011; 11:415-25.

32. Gottems LBD, Morais TCP, Gonçalves AC, Ribeiro $\mathrm{D}$, Silva $\mathrm{CR}$, Rodrigues $\mathrm{DS}$, et al. Acesso à rede de atenção à gestação, parto e nascimento na perspectiva das usuárias. Revista Eletrônica Gestão \& Saúde 2015; 6:95-115.

33. Barros FC, Matijasevich A, Requeio JH, Giugliani E, Maranhão EG, Monteiro CA, et al. Recent trends in maternal, newborn, and child health in Brazil: progress toward Millennium Development Goals 4 and 5. Am J Public Health 2010; 100:1877-89.

34. Wagner M. Fish can't see water. The need to humanize birth. Int J Gynecol Obstet 2001; 75 Suppl 1:S25-37.
35. Belo MNM, Azevedo PTACC, Belo MPM, Serva VMSBD, Batista Filho M, Figueiroa JN. Aleitamento materno na primeira hora de vida em um Hospital Amigo da Criança: prevalência, fatores associados e razões para sua não ocorrência. Rev Bras Saúde Matern Infant 2014; 14:65-72.

36. Moreira MEL, Gama SGN, Pereira APE, Silva AAM, Lansky S, Pinheiro RS, et al. Práticas de atenção hospitalar ao recém-nascido saudável no Brasil. Cad Saúde Pública 2014; 30 Suppl 1:S128-39.

37. Tesser CD, Knobel R, Andrezzo HFA, Diniz SG. Violência obstétrica e prevenção quaternária: o que é e o que fazer. Rev Bras Med Fam Comunidade 2014; 10:1-12.

38. Figueredo MS, Silva RAR, Oliveira DKMA, Vieira NRS, Costa DARS, Davim RMB. Grau de satisfação de puérperas quanto à qualidade da assistência no alojamento conjunto de uma maternidade pública. Rev Pesqui Cuid Fundam (Online) 2015; 7:2697-706. 


\section{Abstract}

The study assessed the quality of care for labor and childbirth in the public hospital system in the city of Recife, Pernambuco State, Brazil, according to the mothers' satisfaction, using a cross-sectional exploratory study in all the public hospitals in the network, grouped according to type of hospital management and interviews with 1,000 mothers. Quality of care was measured according to management strata and the following dimensions of quality: reception; respect for individual rights; prenatal and childbirth care; and ambience. The results' significance was analyzed with the Pearson and Friedman chi-square test. There was high prenatal coverage, not linked to childbirth care, and intense migration of deliveries. Waiting time for care by the healthcare team was long, the proportion of vaginal deliveries in the municipal maternity services was $80 \%$, and only $16 \%$ of the deliveries included skin-to-skin contact with the newborn; breastfeeding occurred in the birthing room in $11 \%$ of the deliveries. Among the various management modalities, the charitable hospital rated highest. The target dimension showed significant differences, with higher satisfaction rates for the following: respect (88.2\%), kindness (86.7\%), physicians' work (85.2\%), and trust in the healthcare staff (84.3\%). The highest rates of dissatisfaction were for: temperature on the ward (62.2\%), possibility of lodging complaints (48.1\%), quantity and quality of hospital clothing and bedding (49.2\%), and privacy (43\%). Despite positive strides, the findings show the need for reorganization of obstetric care policy, with regionalization, regulation, consolidation of networks of care, and interventions in the healthcare setting, aimed at consolidating the humanization of care.

Health Evaluation; Quality of Health Care; Parturition

\section{Resumen}

El estudio evaluó la calidad de la atención al parto en la red pública hospitalaria, en la ciudad de Recife, Pernambuco, Brasil, según la satisfacción de las gestantes, mediante un estudio transversal de cuño exploratorio, en todas las unidades públicas hospitalarias de la red, agrupadas en naturaleza de la gestante, por la auscultación de 1.000 gestantes. La calidad de la atención se realizó según estratos de gestión y dimensiones de la calidad: acogida; respeto a los derechos individuales; asistencia prenatal y parto; y entorno. La significancia de los resultados se analizó por el test chi-quadrado de Pearson y Friedman. Hubo una alta cobertura del prenatal, sin vinculación con la asistencia al parto e intensa migración de partos. El tiempo de espera para ser atendido por el equipo fue largo, la proporción de partos vaginales fue superior en las maternidades municipales (80\%), hubo en sólo un $16 \%$ de los partos un contacto piel a piel con el bebé; la lactancia tuvo lugar en un $11 \%$ en la sala de parto. Entre las esferas de gestión, el hospital filantrópico tuvo una mejor evaluación. Las dimensiones evaluadas presentaron diferencias significativas, existiendo mayores satisfacciones con los aspectos: respeto (88,2\%), amabilidad (86,7\%), trabajo de los médicos $(85,2 \%)$ y confianza en los profesionales $(84,3 \%)$. Respecto a las mayores insatisfacciones se relacionaron con: temperatura de la enfermería $(62,2 \%)$, posibilidad de realizar reclamaciones $(48,1 \%)$, cantidad y calidad de la ropa $(49,2 \%)$ y privacidad (43\%). A pesar de las conquistas, los hallazgos revelaron la necesidad de reorganización de la política de asistencia obstétrica con la regionalización, regulación, consolidación de las redes de atención e intervenciones en el entorno, con el fin de consolidar la humanización de la atención.

Evaluación en Salud; Calidad de la Atención de Salud; Parto
Recebido em 10/Out/2016

Versão final reapresentada em 21/Fev/2017 Aprovado em 08/Mar/2017 